\title{
ON GENERALIZED COMPLETE METRIC SPACES
}

\author{
BY CALVIN F. K. JUNG
}

Communicated by J. B. Diaz, September 19, 1968

1. Introduction. In reference [3], Luxemburg defined the notion of generalized complete metric space as follows.

Definition. The pair $(X, d)$ is called a generalized complete metric space if $X$ is a nonvoid set and $d$ is a function from $X \times X$ to the extended reals satisfying the following conditions:

(D0) $d(x, y) \geqq 0$,

(D1) $d(x, y)=0$ if and only if $x=y$,

(D2) $d(x, y)=d(y, x)$,

(D3) $d(x, y) \leqq d(x, z)+d(z, y)$,

(D4) every $d$-Cauchy sequence in $X$ is $d$-convergent, i.e., if $\left\{x_{n}\right\}$ is a sequence in $X$ such that $\lim _{m, n \rightarrow \infty} d\left(x_{n}, x_{m}\right)=0$, then there is an $x \in X$ with $\lim _{n \rightarrow \infty} d\left(x_{n}, x\right)=0$.

Some fixed point theorems of the alternative for contractions on such spaces had been proved which include, as a special case, the fixed point theorem of Banach for contractions on complete metric spaces (see [1]). For further information and references, see references [2] and [4].

For convenience, we shall call a pair $(X, d)$ a generalized metric space if all but condition $\mathrm{D} 4$ of the above definition are satisfied.

Let $\left\{\left(X_{\alpha}, d_{\alpha}\right) \mid \alpha \in Q\right\}$ be a family of disjoint metric spaces. Then there is a natural way of obtaining a generalized metric space $(X, d)$ from $\left\{\left(X_{\alpha}, d_{\alpha}\right) \mid \alpha \in Q\right\}$ in the following manner. Let $X$ be the union of $\left\{X_{\alpha} \mid \alpha \in a\right\}$. For any $x, y \in X$, define

$$
\begin{aligned}
& d(x, y)=d_{\alpha}(x, y) \quad \text { if } x, y \in X_{\alpha} \quad \text { for some } \alpha \in \propto, \\
& =+\infty \quad \text { if } x \in X_{\alpha}, y \in X_{\beta} \text { for some } \alpha, \beta \in Q \text { with } \alpha \neq \beta \text {. }
\end{aligned}
$$

Clearly $(X, d)$ is a generalized metric space. Moreover, if each $\left(X_{\alpha}, d_{\alpha}\right)$ is also complete, then $(X, d)$ is a generalized complete metric space. The main purpose of this paper is to show that the above procedure is the only way to obtain generalized (complete) metric spaces (see $\S 2$. Consequently, most of the fixed points theorems of the alternative on such spaces can be obtained from the corresponding fixed point theorems on metric spaces (see $\$ 3$ ).

2. Decomposition. Let $(X, d)$ be a generalized metric space. Define a relation $\sim$ on $X$ as follows.

$x \sim y$ if and only if $d(x, y)<+\infty$. Then $\sim$ is obviously an equiva. 
lence relation on $X$ and, therefore, $X$ is decomposed uniquely into (disjoint) equivalence classes $X_{\alpha}, \alpha \in Q$. We shall call this decomposition of $X$ the canonical decomposition, for convenience.

THEOREM. Let $(X, d)$ be a generalized metric space. $X=U\left\{X_{\alpha} \mid \alpha \in a\right\}$ the canonical decomposition and

$$
d_{\alpha}=\left.d\right|_{x_{\alpha} \times x_{\alpha}}
$$

for each $\alpha \in a$. Then

(a) for each $\alpha \in Q,\left(X_{\alpha}, d_{\alpha}\right)$ is a metric space,

(b) for any $\alpha, \beta \in Q$ with $\alpha \neq \beta$,

$$
d(x, y)=+\infty
$$

for any $x \in X_{\alpha}$ and $y \in X_{\beta}$,

(c) $(X, d)$ is a generalized complete metric space if and only if, for each $\alpha \in Q,\left(X_{\alpha}, d_{\alpha}\right)$ is a complete metric space.

Proof. Parts (a) and (b) are clear.

(c) Suppose that $(X, d)$ is a generalized complete metric space and, for any $\alpha \in a$, let $\left\{x_{n}\right\}$ be a $d_{\alpha}$-Cauchy sequence in $X_{\alpha}$. Then $\left\{x_{n}\right\}$ is a $d$-Cauchy sequence in $X$ and, therefore, is $d$-convergent to $x \in X$. Since clearly limits of sequences are unique and since $d\left(x_{n}, x\right)$ $<+\infty$ for sufficiently large $n, x \in X_{\alpha}$ and the sequence $\left\{x_{n}\right\}$ is $d_{\alpha^{-}}$ convergent to $x$.

Conversely, suppose that, for each $\alpha \in Q,\left(X_{\alpha}, d_{\alpha}\right)$ is a complete metric space and let $\left\{x_{n}\right\}$ be a $d$-Cauchy sequence in $X$. There exists a positive integer $N$ such that $d\left(x_{n}, x_{m}\right)<+\infty$ for $m, n \geqq N$. Hence there exists an $\alpha \in Q$ such that $x_{n} \in X_{\alpha}$ for $n \geqq N$ and the sequence $\left\{x_{n} \mid n \geqq N\right\}$ is $d_{\alpha}$-Cauchy in $X_{\alpha}$. Thus $\left\{x_{n} \mid n \geqq N\right\}$ is $d_{\alpha}$-convergent to $x \in X_{\alpha}$. Clearly $\left\{x_{n}\right\}$ is $d$-convergent to $x$ in $X$.

\section{Fixed points theorem.}

3.1. TheOREM. Let $(X, d)$ be a generalized metric space,

$$
X=U\left\{X_{\alpha} \mid \alpha \in a\right\}
$$

the canonical decomposition and let

$$
T: X \rightarrow X
$$

be a mapping such that

$$
d(T(x), T(y))<+\infty
$$

whenever $x, y \in X$ and $d(x, y)<+\infty$. Then $T$ has a fixed point if and only if 


$$
T_{\alpha}=\left.T\right|_{X_{\alpha}}: X_{\alpha} \rightarrow X_{\alpha}
$$

has a fixed point for some $\alpha \in Q$.

Since the proof is clear, we merely remark that the condition $\left(^{*}\right)$ is necessary for $T_{\alpha}$ to be a mapping from $X_{\alpha}$ into $X_{\alpha}$. Furthermore, a "local" version of Theorem 3.1 can be obtained as follows.

3.2. TheOREM. Let $(X, d)$ be a generalized metric space,

$$
X=\bigcup\left\{X_{\alpha} \mid \alpha \in a\right\}
$$

the canonical decomposition and let

$$
T: X \rightarrow X
$$

be a mapping. If there exists a constant $r>0$ such that

$$
d(T(x), T(y))<r
$$

whenever $d(x, y)<r$, then $T$ has a fixed point if and only if for some subset $Y \subset X$ of diameter (defined in the usual manner) $2 r$, the restriction

$$
\left.T\right|_{Y}: Y \rightarrow Y
$$

has a fixed point.

4. Applications. As illustrative applications, we shall prove a theorem which easily implies the main theorem of [2].

TheOREM. Let $(X, d)$ be a generalized complete metric space,

$$
X=\bigcup\left\{X_{\alpha} \mid \alpha \in a\right\}
$$

the canonical decomposition and let

$$
T: X \rightarrow X
$$

be a contraction, i.e., there is a constant $q$ such that $0 \leqq q<1$ and that

$$
d(T(x), T(y)) \leqq q d(x, y)
$$

for all $x, y \in X$. If there exists an $x_{0} \in X$ such that $d\left(x_{0}, T\left(x_{0}\right)\right)<+\infty$, then for some $\alpha \in a$, the restriction

$$
T_{\alpha}=\left.T\right|_{x_{\alpha}}: X_{\alpha} \rightarrow X_{\alpha}
$$

is a contraction.

Proof. Since $d\left(x_{0}, T\left(x_{0}\right)\right)<+\infty$, both $x_{0}$ and $T\left(x_{0}\right)$ belong to the same $X_{\alpha_{0}}$, for some $\alpha_{0} \in a$. Since $T$ is contractive, $T\left(X_{\alpha_{0}}\right) \subset X_{\alpha_{0}}$ and the restriction $T_{\alpha_{0}}=\left.T\right|_{\alpha_{\alpha_{0}}}$ is a contraction from $X_{\alpha_{0}}$ into $X_{\alpha_{0}}$. 
Corollary. Assuming the same hypotheses as in the theorem, let $x \in X$ and consider the "sequence of successive approximations with initial element $x$,"

$$
x, T x, T^{2} x, \cdots, T^{l} x, \cdots .
$$

Then the following alternative holds: either

(A) for every integer $l=0,1,2, \cdots$, one has

$$
d\left(T^{l} x, T^{l+1} x\right)=+\infty
$$

or

(B) the sequence $x, T x, T^{2} x, \cdots, T^{2} x, \cdots$, is $d$-convergent to a fixed point of $T$.

Proof. If (A) does not hold, then for some $l, d\left(T^{l} x, T^{l+1} x\right)<+\infty$. Letting $x_{0}=T^{l} x$, the theorem shows that

$$
T_{\alpha}=\left.T\right|_{x_{\alpha}}: X_{\alpha} \rightarrow X_{\alpha}
$$

is a contraction, where $X_{\alpha}$ is the complete metric space containing $x_{0}$. By the fixed point theorem of Banach for contractions on complete metric spaces, the sequence $T^{l} x, T^{l+1} x, \ldots$ is $d_{\alpha}$-convergent to a fixed point $p$ of $T_{\alpha}$. This implies that (B) holds.

\section{REFERENCES}

1. S. Banach, Sur les operations dans les ensembles abstraits et leur application aux equations integrales (Doctoral Thesis, University of Lwow), Fund. Math. 3 (1922), 133-181.

2. J. B. Diaz and B. Margolis, A fixed point theorem of the alternative for contractions on a generalized complete metric space, Bull. Amer. Math. Soc. 74 (1968), 305-309.

3. W. A. J. Luxemburg, On the convergence of successive approximations in the theory of ordinary differential equations. II, Nederl. Akad. Wetensch. Proc. Ser. A 61 =Indag. Math. 20 (1958), 540-546.

4. B. Margolis, On some fixed points theorems in generalized complete metric spaces, Bull. Amer. Math. Soc. 74 (1968), 275-282.

Kansas State University, Manhattan, Kansas 66502 